[0212-7199 (2005) 22: 4; pp 162-166] ANALES DE MEDICINA INTERNA Copyright $($ C 2005 ARAN EDICIONES, S.L.

AN. MED. InTERnA (Madrid) Vol. 22, N. ${ }^{\circ} 4$, pp. 162-166, 2005

\title{
Epidemiología del carcinoma hepatocelular en un área rural. Papel de los virus hepatotropos en la supervivencia
}

\author{
F. F. RODRÍGUEZ-VIDIGAL, M. J. BAZ', J. ROMERO, M. DEL PUERTO \\ Servicio de Medicina Interna. 'Servicio de Análisis Clínicos. Hospital de Llerena. \\ Llerena. Badajoz
}

EPIDEMIOLOGY OF HEPATOCELLULAR CARCINOMA IN A RURAL AREA. ROLE OF HEPATOTROPHIC VIRUSES ON SURVIVAL

\section{RESUMEN}

Introducción: Las hepatitis crónicas $\mathrm{C}$ y B son las principales causas de carcinoma hepatocelular (CHC) en el mundo. No se sabe si la infección crónica por los virus de la hepatitis $\mathrm{C}$ (VHC) o B (VHB) es un factor pronóstico para el CHC. El presente estudio busca conocer la epidemiología del CHC en un área de salud rural y valorar si la infección crónica por los VHC o VHB tiene algún impacto en la supervivencia tras el diagnóstico de $\mathrm{CHC}$.

Material y métodos: Estudio retrospectivo de 51 pacientes consecutivos, diagnosticados de CHC entre enero de 1994 y diciembre de 2002 en un Hospital de Primer Nivel. Se analizaron las siguientes variables: edad, sexo, infección por VHC y VHB, abuso crónico de alcohol (toma diaria mayor de $80 \mathrm{~g}$ ), presentación clínica, estadio de Child, número de nódulos hepáticos, tratamiento y supervivencia.

Resultados: La edad media al diagnóstico de CHC fue de 68,5 años de edad (rango 45-90) y 45 pacientes $(88,6 \%$ ) eran varones. Los factores etiológicos más prevalentes fueron la ingestión excesiva de alcohol (66\%) y la infección crónica por el VHC (42,8\%). La infección crónica por el VHB se halló en el 11,9\%. En el 48,9\% de los casos se detectó infección crónica por el VHC o por el VHB. El 25\% estaban asintomáticos y el $66 \%$ en estadio A de Child. La proporción lesión única / CHC multinodular fue 52/48. Únicamente el $6 \%$ de los pacientes pudo recibir tratamiento con intención curativa. La supervivencia media fue de 10,9 \pm 9,1 meses, y no hubo diferencias respecto a la edad, sexo, estadio de Child y número de nódulos hepáticos. La supervivencia fue significativamente mayor en los pacientes con infección crónica por el VHC o por el VHB $(16,7 \pm 13,1$ frente a 4,75 $\pm 5,3$ meses en seronegativos; $\mathrm{p}=0.02)$. En el análisis multivariante, sólo la infección crónica por el VHC o por VHB se asoció con una supervivencia superior a 10 meses (OR 22,3; IC 95\% 1,8-277,9).

Conclusiones: En nuestro medio, los factores etiológicos más prevalentes del CHC son el etilismo y la infección por el VHC. En pacientes con $\mathrm{CHC}$, la infección crónica por el VHC o por el VHB se asocia a una mayor supervivencia.

PALABRAS CLAVE: Carcinoma hepatocelular. Virus de la hepatitis C. Virus de la hepatitis B. Supervivencia.

\begin{abstract}
Background: Chronic hepatitis $C$ and $B$ are the main causes of hepatocellular carcinoma (HCC) worldwide. It is not clear whether chronic hepatitis $C$ or $B$ virus ( $H C V$ or $H B V$ ) infection is a prognostic factor for HCC. This study aimed to asses epidemiology of HCC in a rural area and to determine if chronic $H C V$ or $H B V$ infection had any impact on survival after the diagnosis of $H C C$.

Material and methods: Fifty-one consecutive patients were retrospectively studied. All of them were diagnosed of HCC between january 1994 and december 2002 in a First Level Hospital. The following variables were analysed: age, sex, $\mathrm{HCV}$ and $\mathrm{HBV}$ infection, chronic alcohol abuse (daily intake upper $80 \mathrm{~g}$ ), clinical presentation, Child stage, number ol liver nodules, therapeutic options and survival.

Results: The mean age at diagnosis of HCC was 68,5 years old (age range 45-90) and 45 patients $(88,6 \%)$ were male. Heavy alcohol intake (66\%) and chronic HCV infection $(42,8 \%)$ were the most prevalent etiologic factors. Chronic HBV was found in 11,9\%. Chronic HCV or HBV infection was present in 48,9\%. Twenty-five percent were asymptomatic and $66 \%$ were in Child stage A. The rate single lesion / multilobular HCC was 52/48. Only $6 \%$ of all patients could be treated with a curative intention. The mean survival was 10,9 $\pm 9,1$ months, and there were no differences in age, sex, Child stage and number of nodules. There was a significantly higher survival in patients with chronic HCV or HBV infection $(16,7 \pm 13,1$ months versus $4,75 \pm 5,3$ months in seronegative patients; $p=0.02)$. On multivariate analysis, only chronic HCV or $H B V$ infection was associated with survival longer than 10 months (OR 22,3; CI 95\% 1,8-277,9).

Conclusions: In our area, heavy alcohol abuse and HCV infection were the most prevalent etiologic factors of $\mathrm{HCC}$. Chronic HCV or HBV infection was associated with longer survival in patientes with HCC.
\end{abstract}

KEY WORDS: Hepatocellular carcinoma. Hepatitis C virus. Hepatitis $B$ virus. Survival.

Rodríguez-Vidigal FF, Baz MJ, Romero J, del Puerto M. Epidemiología del carcinoma hepatocelular en un área rural. Papel de los virus hepatotropos en la supervivencia. An Med Interna (Madrid) 2005; 22: 162-166.

Trabajo aceptado: 9 de diciembre de 2004

Correspondencia: Francisco Félix Rodríguez Vidigal. Servicio de Medicina Interna. Hospital de Llerena. Ctra. Badajoz-Granada, s/n. 06900 Llerena. Badajoz. e-mail: franciscof.rodriguez@hllz.ses.juntaex.es 


\section{INTRODUCCIÓN}

El carcinoma hepatocelular $(\mathrm{CHC})$ es la quinta neoplasia más frecuente en el mundo; cada año se diagnostican, según el país, entre 2,6 y 35,5 casos por 100.000 habitantes y su incidencia se halla en franco aumento (1-6). En aproximadamente el $90 \%$ de los casos se desarrolla en pacientes aquejados de cirrosis hepática y los factores asociados más fuertemente con su aparición son el virus de la hepatitis C (VHC), el virus de la hepatitis B (VHB) y el abuso de bebidas alcohólicas (1,7-10).

En la actualidad, además de la resección quirúrgica y de la ablación percutánea, el mejor tratamiento del CHC es el trasplante hepático, pero no resulta una opción terapéutica de fácil acceso dado el amplio desfase entre la elevada demanda y la escasez de donantes $(9,11,12)$. Menos del $10 \%$ de los pacientes a los que se diagnostica un $\mathrm{CHC}$ sobreviven cinco años (13).

Se han descrito diversos factores pronósticos del CHC: un diagnóstico precoz y la posibilidad de tratamiento quirúrgico o ablativo son factores pronósticos positivos, mientras que niveles de alfafetoproteina elevados, la afectación multicéntrica y la presencia de trombosis venosa portal se han asociado a una evolución peor $(7,14,15)$. En lo referente a la influencia de la infección por virus hepatotropos en la supervivencia, en un grupo de pacientes con $\mathrm{CHC}$ que recibieron tratamiento quirúrgico, Ahmad et al (16) detectaron una menor supervivencia libre de enfermedad en aquellos que presentaban infección por el VHC o por el VHB. Por otro lado, Yamanaka et al (17) encuentran una menor supervivencia en sujetos con cirrosis etílica seropositivos para el VHB que en seropositivos para el VHC. Otros autores no hallan influencia de la etiología del CHC en la supervivencia (18).

El objetivo del presente estudio fue conocer las características epidemiológicas del $\mathrm{CHC}$ en un área de salud con bajo acceso al trasplante hepático (12), y valorar si la infección por virus hepatotropos tiene alguna influencia en el pronóstico del $\mathrm{CHC}$.

\section{MATERIAL Y MÉTODOS}

\section{PACIENTES Y CRITERIOS DE INCLUSIÓN}

De modo retrospectivo, se recogieron 51 pacientes consecutivos, diagnosticados de CHC entre enero de 1994 y diciembre de 2002, en el Servicio de Medicina Interna del Hospital de Llerena, un centro de primer nivel localizado en la comarca suroeste de Extremadura, y que atiende a una población de 104.000 habitantes. El diagnóstico de CHC se basó en los hallazgos de dos técnicas de imagen coincidentes o en la combinación de una técnica de imagen y unos niveles de alfafetoproteína superiores a $400 \mathrm{ng} / \mathrm{ml}$, según los criterios de la conferencia EASL de Barcelona (19). En el 50\% de los casos se contó con el criterio diagnóstico citohistológico. En cada paciente se recogieron los siguientes datos: edad, sexo, infección por el VHC (anti-VHC, detectado por enzimoinmunoanálisis y confirmado mediante inmunoblot recombinante), infección por el VHB (HBsAg positivo), abuso crónico de etanol previo (consumo superior a 80 gramos/día), presentación clínica, estadio de la cirrosis de acuerdo con la clasificación de Child-Pugh (20), alfafetoproteína sérica (valor normal inferior a $15 \mathrm{ng} / \mathrm{ml}$ ), número de nódulos hepáticos, tratamien- to y supervivencia. De acuerdo con el número de nódulos se establecieron dos categorías: único y múltiple (en esta última categoría se incluía también al CHC difuso). Los datos se obtuvieron mediante la revisión de las historias clínicas (ingresos y revisiones en consultas) y la detección del último procedimiento diagnóstico o terapéutico llevado a cabo en el hospital o en los centros de salud del área. Se garantizó la inclusión de todos los pacientes posibles mediante búsqueda en la base de datos de informes de alta del Servicio de Medicina Interna y en el registro hospitalario del Conjunto Mínimo Básico de Datos.

\section{ANÁLISIS ESTADÍSTICO}

Para comparar variables cuantitativas se utilizó la prueba de Mann-Whitney y el análisis de la varianza; para las variables categóricas se empleó la prueba de la chi-cuadrado con la corrección de Yates y la prueba exacta de Fisher cuando fue preciso. Se consideró significativo un valor de $p<0,05$. Los factores independientes de la supervivencia se evaluaron de acuerdo con un análisis de regresión logística múltiple (21). Las variables incluidas en el modelo fueron: edad, alfafetoproteina elevada, nódulo único/múltiple, estadio de ChildPugh, abuso de etanol e infección por VHC o por VHB. Todos los análisis estadísticos se llevaron a cabo con la versión 5.0 de StatView for Windows SAS Institute Inc (1992-1998).

\section{RESULTADOS}

\section{DATOS DEMOGRÁFICOS Y EPIDEMIOLÓGICOS}

Dado que el único centro hospitalario del área de salud entre 1994 y 2002 era el Hospital de Llerena, se puede estimar una incidencia de 5,45 nuevos casos de CHC por 100.000 habitantes y año. En el momento del diagnóstico de $\mathrm{CHC}$, la edad media fue de 68,5 $\pm 8,2$ años (rango 45-90). Seis pacientes $(12 \%)$ tenían menos de 60 años, $20(39 \%)$ entre 60 y 69 años, $22(43 \%)$ entre 70 y 79, y tres $(6 \%)$ tenían 80 o más años. El único paciente menor de 50 años estaba coinfectado por el VHC y por el virus de la inmunodeficiencia humana. Predominaron los varones $(88,6 \%)$. Respecto a los factores etiológicos del CHC, el abuso de etanol fue el más prevalente (66\%), seguido de la infección crónica por el VHC $(42,8 \%)$ y de la infección crónica por el VHB (11,9\%). Otros factores etiológicos fueron la presencia de hemocromatosis (en dos pacientes) y la cirrosis de causa autoinmune en un caso (Tabla I). El consumo de alcohol se asoció a infección por VHC o por VHB en el $27 \%$ de los casos y existía hepatitis crónica por alguno de los virus hepatotropos en el $48,9 \%$. Desde que se detectó la infección por VHC o por VHB hasta el momento del diagnóstico de CHC transcurrió una media de 44,5 \pm 94,1 meses (rango 0-336); en siete pacientes se diagnosticó la infección por virus hepatotropo (seis VHC, uno VHB) simultáneamente al diagnóstico de $\mathrm{CHC}$.

\section{DIAGNÓSTICO Y TRATAMIENTO}

Los síntomas de presentación más frecuentes fueron: ascitis y/o edema de miembros inferiores $(36,4 \%)$, dolor abdomi- 
TABLA I

\section{DATOS DEMOGRÁFICOS, EPIDEMIOLÓGICOS Y CLÍNICOS DE LOS PACIENTES CON CHC}

\begin{tabular}{|c|c|c|}
\hline Variable & desviación estándar & $\%$ \\
\hline Edad (años) & $68,5 \pm 8,2$ & \\
\hline \multicolumn{3}{|l|}{ Distribución de edades } \\
\hline$<60$ años & & 12 \\
\hline 60-69 años & & 39 \\
\hline 70-80 años & & 43 \\
\hline$>80$ años & & 6 \\
\hline Sexo masculino & & 88,6 \\
\hline \multicolumn{3}{|l|}{ Factores etiológicos } \\
\hline Alcohol & & 66 \\
\hline VHC & & 42,8 \\
\hline VHB & & 11,9 \\
\hline Otros & & 5,9 \\
\hline \multicolumn{3}{|l|}{ Sintomatología } \\
\hline Asintomático & & 25 \\
\hline Ascitis y/o edemas & & 36,4 \\
\hline Dolor abdominal & & 27,3 \\
\hline Astenio y/o pérdida de peso & & 18,6 \\
\hline Diagnóstico clínico-ecográfico de cirrosis & & 96 \\
\hline \multicolumn{3}{|l|}{ Estadio Child-Pugh } \\
\hline$A$ & & 66 \\
\hline B & & 22 \\
\hline C & & 12 \\
\hline \multicolumn{3}{|l|}{ Alfafetoproteína sérica } \\
\hline Normal $(<15 \mathrm{ng} / \mathrm{ml})$ & & 33,3 \\
\hline $16-399 \mathrm{ng} / \mathrm{ml}$ & & 33,3 \\
\hline$>400 \mathrm{ng} / \mathrm{ml}$ & & 33,3 \\
\hline \multicolumn{3}{|l|}{ Morfología del $\mathrm{CHC}$} \\
\hline Único & & 52 \\
\hline Multinodular o difuso & & 48 \\
\hline \multicolumn{3}{|l|}{ Tratamiento } \\
\hline Resección quirúrgica & & 2 \\
\hline Etanolización & & 3,9 \\
\hline Quimioembolización & & 9,8 \\
\hline Sintomático & & 84 \\
\hline
\end{tabular}

nal $(27,3 \%)$ y astenia y/o pérdida de peso $(18,6 \%)$. En el $25 \%$ de los casos, el paciente se encontraba asintomático y el CHC se diagnosticó a partir de anomalías en un estudio analítico o ecográfico. Con excepción de dos pacientes, en todos existían signos laparoscópicos, histológicos o clínico-radiológicos de cirrosis hepática (96\%). Predominaron los sujetos en estadio A de Child-Pugh en el momento del diagnóstico (66\%); 22\% se encontraban en un estadio B y $12 \%$ en estadio C. La alfafetoproteina sérica se hallaba dentro de los límites normales en la tercera parte de los enfermos, entre 16 y $339 \mathrm{ng} / \mathrm{ml}$ en otro tercio y era superior a $400 \mathrm{ng} / \mathrm{ml}$ en el tercio restante. La proporción CHC único/CHC multicéntrico o difuso fue de 52/48. Únicamente en tres pacientes existía un nódulo único menor de $40 \mathrm{~mm}$ de diámetro. El tratamiento aplicado fue: resección quirúrgica (un caso), etanolización (dos casos), quimioembolización (cinco casos) y, en los casos restantes, medidas sintomáticas. Ningún paciente pudo recibir trasplante hepático (Tabla I).

\section{SUPERVIVENCIA}

El 60\% de los pacientes diagnosticados de CHC sobrevivió menos de un año y el $20 \%$ más de dos años. La supervivencia media fue de 10,9 \pm 9,1 meses (rango 0-24) y no se encontraron diferencias significativas con respecto a la edad, sexo, consumo de alcohol, valor de alfafetoproteina sérica, número de nódulos hepáticos y estadio Child-Pugh. Se detectó una mayor supervivencia en pacientes con hapatitis crónica por VHC o por VHB que en seronegativos $(16,7 \pm 13,1$ meses frente a 4,75 $\pm 5,3$ meses, $\mathrm{p}=0,02$ ). Por otro lado, el tiempo transcurrido desde que se conocía la infección por el VHC o VHB hasta el diagnóstico de CHC, no se correlacionó con la supervivencia (Tablas II y III). Los resultados del análisis de regresión logística mostraron que la hepatitis crónica por

TABLA II

SUPERVIVENCIA DEL CHC Y VARIABLES CUALITATIVAS

\begin{tabular}{|c|c|c|}
\hline Variable & Supervivencia (meses) & $p$ \\
\hline Sexo & & NS \\
\hline Varón & & $13,5 \pm 12,1$ \\
\hline Mujer & & $6,0 \pm 5,7$ \\
\hline Abuso de alcohol & & NS \\
\hline Sí & & $14,6 \pm 14,2$ \\
\hline No & & $10,3 \pm 9,2$ \\
\hline Número de nódulos & & NS \\
\hline Único & & $16,6 \pm 15,8$ \\
\hline Múltiple o difuso & & $10,5 \pm 10,0$ \\
\hline Hepatitis crónica por VHC o VHC & & $<0,03$ \\
\hline Sí & & $16,7 \pm 13,1$ \\
\hline No & & $4,7 \pm 5,3$ \\
\hline
\end{tabular}

TABLA III

CARACTERÍSTICAS DE LOS PACIENTES CON CHC Y SUPERVIVENCIA

\begin{tabular}{|c|c|c|c|}
\hline Variable & $\begin{array}{c}\text { Supervivencia } \\
>10 \text { meses }\end{array}$ & $\begin{array}{c}\text { Supervivencia } \\
<10 \text { meses }\end{array}$ & $p$ \\
\hline Edad (años)* & $65,5 \pm 10,2$ & $68,5 \pm 5,0$ & NS \\
\hline Sexo varón & $92 \%$ & $92 \%$ & NS \\
\hline \multicolumn{4}{|l|}{ Alfafetoproteína sérica } \\
\hline$>15 \mathrm{ng} / \mathrm{ml}$ & & & NS \\
\hline Sí & $67 \%$ & $73 \%$ & \\
\hline No & $33 \%$ & $27 \%$ & \\
\hline Abuso de etanol & & & NS \\
\hline Sí & $54 \%$ & $67 \%$ & \\
\hline No & $46 \%$ & $33 \%$ & \\
\hline Número de nódulos & & & NS \\
\hline Único & $50 \%$ & $36 \%$ & \\
\hline Múltiple o difuso & $50 \%$ & $64 \%$ & \\
\hline Hepatitis crónica VHC o VHB & & & $<0,007$ \\
\hline Sí & $92 \%$ & $42 \%$ & \\
\hline No & $8 \%$ & $58 \%$ & \\
\hline Estadio Child-Pugh & & NS & \\
\hline A & $56 \%$ & $42 \%$ & \\
\hline $\mathrm{B} \circ \mathrm{C}$ & $43 \%$ & $57 \%$ & \\
\hline
\end{tabular}

NS: no significativo. ${ }^{*}$ Valor expresado en media \pm desviación estándar 
VHC o VHB era un factor asociado de modo independiente con una supervivencia superior a 10 meses (odds ratio 22,3; intervalo de confianza del 95\% 1,8-277,9). No se encontró asociación con otras variables incluidas en el sistema (edad, abuso de etanol, alfafetoproteína elevada, nódulo único).

\section{DISCUSIÓN}

En el presente estudio se encuentra que los factores etiológicos de CHC más prevalentes en nuestro área de salud son el abuso de alcohol y la infección crónica por virus hepatotropos, especialmente el VHC. Por otro lado, en los pacientes diagnosticados de $\mathrm{CHC}$, la infección por $\mathrm{VHC}$ o por $\mathrm{VHB}$ se asocia con una mayor supervivencia.

La incidencia media anual de CHC en el área, durante el período de tiempo estudiado, fue de 5,45 nuevos casos por 100.000 habitantes, en consonancia con los datos que se observan en otras regiones españolas y europeas, por encima de la incidencia observada en Estados Unidos (3,1/100.000) y muy por debajo de los 35 casos $/ 100.000$ habitantes que se recogen en el sudeste asiático $(3,4)$. La mayor parte de los pacientes tenían entre 60 y 80 años en el momento del diagnóstico y la edad media (68,5 años) fue superior a la observada en Japón (52 años) e Italia (60 años) y similar a la detectada en Estados Unidos $(4,8,15)$. Respecto a los factores etiológicos del $\mathrm{CHC}$, al igual que en otras series, con frecuencia se asoció el abuso de etanol con las hepatitis crónicas de etiología vírica y predominó la infección por el VHC sobre la infección por el VHB, si bien la proporción de casos seropositivos para el VHC $(42,8 \%)$ fue menor que la hallada en otras zonas de España y en otros países mediterráneos (8,22-24). Es preciso mencionar el escaso tiempo transcurrido entre el diagnóstico de la infección por VHC o VHB y el diagnóstico del CHC (media inferior a 4 años), cuando el CHC suele aparecer 20-30 años después de la primoinfección. Dicho dato traduce una deficiente capacidad en detectar precozmente la infección por el VHC, ya recogida con anterioridad (25).

La presencia de datos clínico-radiológicos de cirrosis hepática en casi todos los casos, con frecuencia en estadio A de Child-Pugh, y la escasa sensibilidad de la alfafetoproteina son congruentes con otras series publicadas $(7,8,22)$. Destaca el reducido número de pacientes con nódulos de pequeño tamaño $(6 \%)$ que pudieran recibir tratamiento con intención curativa, en contraste con el $30-40 \%$ que se recoge en otras regiones 11,26. El retraso en el diagnóstico puede haber estado condicionado por la escasa dotación en recursos humanos y en camas hospitalarias $(1,2 / 1000$ habitantes $)$ que hubo en nuestra comarca durante el período de estudio.

Fruto del predominio de $\mathrm{CHC}$ recogidos en fase avanzada es la pobre supervivencia que se alcanza a los dos años (20\%), similar al 16\% encontrado por Llovet et al (13) en un subgrupo de pacientes con $\mathrm{CHC}$ sintomático o con patrón invasivo y por otros estudios europeos $(27,28)$. La supervivencia media de 10,9 meses es superior a la que hallan Petry et al (7) en enfermos con $\mathrm{CHC}$ que recibieron tratamiento sintomático (5,8 meses).

Es llamativo el hallazgo de una supervivencia significativamente mayor en sujetos con datos serológicos de infección por el VHC o por el VHB (media de 16,7 meses) que en seronegativos para ambos virus (media de 4,75 meses) y que la infección por virus hepatotropos sea una variable independiente relacionada con la supervivencia. Los resultados contrastan con los de Gelatti et al (18), que no encontraron influencia de la etiología del $\mathrm{CHC}$ en la supervivencia en una serie de 384 sujetos con CHC (29\% con datos de infección por virus hepatotropos) y con los resultados de Ahmad et al (16), que obtuvieron una asociación de la seropositividad para VHB o VHC con una menor supervivencia libre de enfermedad en una serie de 77 pacientes que recibieron tratamiento quirúrgico para el CHC (80\% con infección por el VHB o por el VHC).

La principal limitación de nuestro estudio es el reducido tamaño de la muestra, si bien representa la mayoría de los diagnósticos de $\mathrm{CHC}$ en un área concreta durante un tiempo en que era atendida por un único centro hospitalario.

Como conclusión cabe reseñar la concordancia de los datos epidemiológicos y clínicos aportados con los publicados previamente, así como la correlación hallada entre la infección por el VHC o por el VHB y una mayor supervivencia, asociación que merece ser investigada en futuros estudios con mayor número de casos. Por otro lado, la escasa proporción detectada de casos de CHC subsidiarios de tratamiento curativo debe conducirnos (a clínicos y a gestores) a implementar medios que mejoren el diagnóstico precoz del CHC.

\section{Bibliografía}

1. Bosch FX, Ribes J, Borras J. Epidemiology of primary liver cancer. Sermin Liver Dis 1999; 19: 271-86

2. Law MG, Roberts SK, Dore GJ, Kaldor JM. Primary hepatocellular carcinoma in Australia, 1978-1997: increasing incidence and mortality. Med J Aust 2000; 173: 403-5

3. Parkin DM, Brey F, Ferlay J, Pirani P. Estimating the world cancer burden. Globocan 2000. Inf J Cancer 2001; 94: 153-6

4. El-Serag HB. Hepatocellular carcinoma and hepatitis C in United States. Hepatology 2002; 36 (Supl. 1): S74-83

5. ElSaadany S, Tepper M, Mao Y, Semenciw R, Giulivi A. An epidemiologic study of hepatocellular carcinoma in Canada. Can J Public Health 2002; 93: 443-6

6. Kiyosawa K, Tanaka E. Characteristics of hepatocellular carcinoma in Japan. Oncology 2002; 62 (Supl. 1): 5-7

7. Petry W, Heintges T, Hensel F, Erhardt A, Wenning M, Niederau C, Haussinger D. Hepatocellular carcinoma in Germany. Epidemiology, etiology, clinical aspects and prognosis in 100 consecutive patients of a

university clinic. Z Gastroenterol 1997; 35: 1059-67

8. Stroffolini T, Andreone P, Andriulli A, Ascione A, Craxi A, Chiaramonte $\mathrm{M}$, et al. Characteristics of hepatocellular carcinoma in Italy. J Hepatol 1998; 29: 944-52

9. Okuda K. Hepatocellular carcinoma. J Hepatol 2000; 32 (Supl. 1): 225237

10. Bosch FX, Ribes J. Epidemiology of liver cancer in Europe. Can J Gastroenterol 2000; 14: 621-30

11. Bruix J, Llovet JM. Prognostic prediction and treatment strategy in hepatocellular carcinoma. Hepatology 2002; 35: 519-24

12. Cuende N, Miranda B, Cañón JF, Naya T, Garrido G. Criterios de priorización para el acceso al trasplante. El caso del trasplante hepático en España. Med Clin (Barc) 2003; 120: 380-6

13. Llovet JM, Bustamante J, Castels A, Vilana R, Ayuso MC, Sala M, et al. Natural history of untreated nonsurgical hepatocellular carcinoma: rationale for the design and evaluation of therapeutic trials. Hepatology 1999; 29: 62-7 
14. Llovet JM, Bru C, Bruix J. Prognosis of hepatocellular carcinoma: the BCLC staging classification. Semin Liver Dis 1999; 19: 329-38

15. Tanizaki H, Ryu M, Kinoshita T, Kawano N, Konishi M, Cho A, et al. Comparison of clinical features and survival in patients with hepatitis B and C virus-related hepatocellular carcinoma. Jpn J Clin Oncol 1997; 27: $67-70$

16. Ahmad SA, Bilimoria MM, Wang X, Izzo F, Delrio P, Marra P, et al. Hepatitis $\mathrm{B}$ or $\mathrm{C}$ virus serology as a prognostic factor in patients with hepatocellular carcinoma. J Gastroenterol Surg 2001; 5: 468-76

17. Yanamaka T, Shiraki K, Nakazawa S, Okano H, Ito K, Deguchi M, et al. Impact of hepatitis B and C virus infection on the clinical prognosis of alcoholic liver cirrhosis. Anticancer Res 2001; 21: 2937-40

18. Gelatti U, Donato F, Tagger A, Fantoni C, Portolani N, Ribero ML, et al. Etiology of hepatocellular carcinoma influences clinical and pathologic features but not patient survival. Am J Gastroenterol 2003; 98: 90714

19. Bruix J, Sherman M, Llovet JM, Beaugrand M, Lencioni R, Burroughs AK, et al. Clinical management of hepatocellular carcinoma. Conclusions of the Barcelona-2000 EASL Conference. J Hepatol 2001; 35: 421-30

20. Pugh RNH, Murria IM, Dawson JL, Pietroni MC, Willians R.Transection of the esophagus in Italy: a multicentric, controlled study. HepatoGastroenterol 1983; 30: 48-50.

21. Kleinbaum DG, Kupper LL, Chambless LE. Logistic regression analy- sis of epidemiological data. Theory and practice. Commun Statist Theory Methods 1982; 71: 485-547

22. Crespo J, Dueñas C, Sánchez-Antolín G, Fábrega E, Carte B, PonsRomero F. Infección por el virus de la hepatitis $\mathrm{C}$ y $\mathrm{C}$ en pacientes con carcinoma hepatocelular. Med Clin (Barc) 1996; 106: 241-5

23. Yu MC, Yuan JM, Govindarajan S, Ross RK. Epidemiology of hepatocellular carcinoma. Can J Gastroenterol 2000; 14: 703-9

24. Hellerbrand C, Hartmann a, Richter G, Knoll A, Wiest R, Scholmerich $\mathrm{J}$, et al. Hepatocellular carcinoma in southern Germany: epidemiological and clinocopathological characteristics and risk farctors. Dig Dis 2001; 19: 345-51

25. Rodríguez-Vidigal FF, Baz MJ, Fernández FJ, Najarro F. Infección por el virus de la hepatitis $\mathrm{C}$ en un hospital de primer nivel de ámbito rural: estudio descriptivo en la década 1991-1999. Enf Infecc Microbiol Clin 2003; $21: 140-4$

26. Llovet JM, Burroughs A, Bruix J. Hepatocellular carcinoma. Lancet 2003; 362: 1907-17

27. Livraghi T, Bolondi L, Buscarini L, Cottone M, Mazzioti A, Morabito A, Torzilli G. No treatment, resection and etanol injection in hepatocellular carcinoma: a retrospective análisis of survival in 391 patients with cirrosis. J Hepatol 1995; 22: 522-6

28. Villa E, Moles A, Ferretti I, Buttafoco P, Grottola A, Del Bruno M, et al. Natural history of inoperable hepatocellular carcinoma: rationale for the design and evaluation of therapeutic trials. Hepatology 1999; 29: 62-7. 